\title{
Author Correction: N-terminomics identifies Prli42 as a membrane miniprotein conserved in Firmicutes and critical for stressosome activation in Listeria monocytogenes
}

Francis Impens, Nathalie Rolhion, Lilliana Radoshevich, Christophe Bécavin, Mélodie Duval, Jeffrey Mellin, Francisco García del Portillo, M. Graciela Pucciarelli, Allison H. Williams and Pascale Cossart

Correction to: Nature Microbiology https://doi.org/10.1038/nmicrobiol.2017.5, published online 13 February 2017.

This Article contains a URL for a publically available whole-genome browser (http://nterm.listeriomics.pasteur.fr). However, due to technical constraint, this website has been replaced with an alternative (https://listeriomics.pasteur.fr).

Published online: 25 June 2018

https://doi.org/10.1038/s41564-018-0197-4 